\title{
Perception and Motion Planning for Pick-and-Place of Dynamic Objects
}

\author{
Anthony Cowley ${ }^{1}$, Benjamin Cohen ${ }^{1}$, William Marshall ${ }^{2}$, Camillo J. Taylor ${ }^{1}$ and Maxim Likhachev ${ }^{3}$
}

\begin{abstract}
Mobile manipulators have brought a new level of flexibility to traditional automation tasks such as tabletop manipulation, but are not yet capable of the same speed and reliability as industrial automation. We present approaches to 3D perception and manipulator motion planning that enable a general purpose robotic platform to recognize and manipulate a variety of objects at a rate of one pick-and-place operation every $6.7 \mathrm{~s}$, and work with a conveyor belt carrying objects at a speed of $33 \mathrm{~cm} / \mathrm{s}$.
\end{abstract}

\section{INTRODUCTION}

Robotics researchers regularly endow robot platforms with new capabilities that increase the breadth of potential applications and push the boundaries of autonomy. In contrast, industrial automation is driven by a pragmatism dictated by the need to optimize throughput and reliability. The hope of both is that, as multi-purpose robotic platforms become more capable, they will be able to take over an increasing fraction of the tasks currently handled by application specific, fixed installation automation, thereby granting all applications greater levels of modularity and adaptability.

We are now seeing an acceleration of the rate at which research robotics feeds into engineering practice. This crossover is arguably being led today by Rethink Robotics's Baxter platform: a movable manipulation platform with many of the characteristics of general purpose research platforms, but sold to industry customers. On the research side, general purpose robotics has taken a large step forward over the past few years, driven in no small part by the development of Willow Garage's ROS software ecosystem, Willow Garage's PR2 mobile manipulation platform, and Microsoft's Kinect sensor that has made RGB-D perception ubiquitous in robot perception.

We apply these technologies to the problem of tabletop manipulation at performance levels relevant to industrial use. Performing this task involves 3D object recognition, pose estimation, and the planning of collision-free manipulator trajectories to first grasp an object, then move it to its destination. We present a high performance, high precision perception and planning combination that enables the PR2 to perform pick-and-place operations on dynamic objects by working with a conveyor belt that drives objects past the robot at a speed of $33 \mathrm{~cm} / \mathrm{s}$.

\footnotetext{
${ }^{1}$ GRASP Laboratory, University of Pennsylvania, \{acowley, bcohen, cjtaylor\}@ seas. upenn.edu

${ }^{2}$ Lehigh University, USA wcm214 alehigh.edu

${ }^{3}$ Robotics Institute, Carnegie Mellon University, maximecs. cmu.edu
}

\section{RELATED WORK}

Factory automation typically relies on careful environment control and predictable inputs. Industrial perception has historically relied on 2D visual sensing where vision sensing is required [1]. This state of affairs is changing as general purpose robots lead the shift to less structured environments, a greater variety of behaviors, and full 3D perception and planning.

Recent approaches to perception for manipulation has moved beyond standard 2D image processing by applying a variety of new sensing modalities to the problem. A multiflash camera sensor has been used to observe depth edges of parts piled in a bin [2]. This work focused on fine manipulation in a highly cluttered environment, and was able to achieve a $94 \%$ success rate with just under one second spent on object pose estimation. Object recognition and pose estimation in complex, noisy scenes is also demonstrated in [3] by relying on an RGB-D sensor.

An example of a responsive general purpose robotic platform interacting with moving objects may be found in [4], in which a mobile humanoid robot is given the ability to catch balls tossed toward it. This application requires great care and attention be given to processing time, as being late is not an option. In this case, the software performs real-time circle detection at $35 \mathrm{~ms}$ per image.

The aforementioned PR2 has been used extensively in tabletop manipulation research [5]. This work focused on reliability and modularity, and has been demonstrated to enable the PR2 to pick up a variety of objects, including those previously unknown to the software.

Raw performance of perception for manipulator planning was a primary consideration in [6], in which a signed truncated distance field computed from Kinect data on a high-end GPU is used to drive manipulator planning. The result is the ability of a manipulation system to execute a planned, collision free path three seconds after first exposure to the scene.

\section{PERCEPTION}

The perception system employed here is made up of a staged processing pipeline based on RGB-D sensor data, and the PR2's own pose estimation, Figure 1. The system is given information about objects of interest at startup, then takes its input from ROS, and publishes localized, identified objects as they are detected. The overall procedure is that table geometry is extracted to perform an initial interpretation of the scene that disregards a large fraction of sensor data. Detected points that lie above the work surface are clustered, and those clusters are recognized and localized to provide 


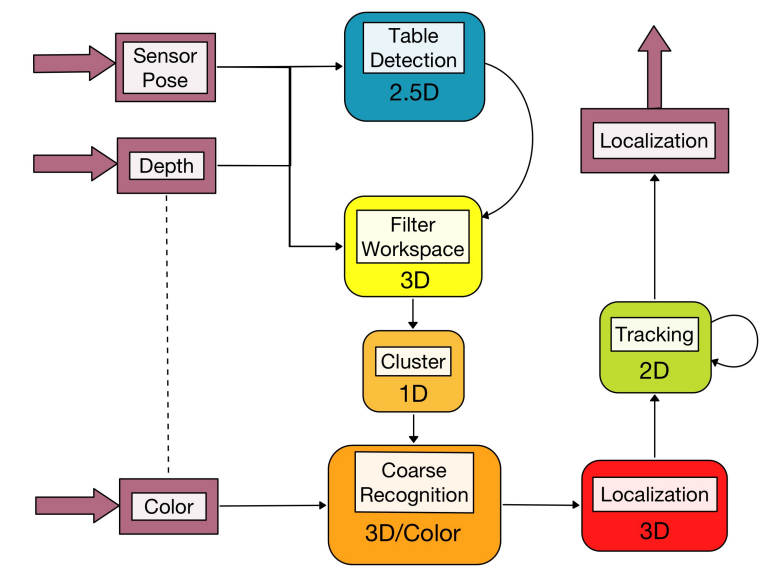

Fig. 1. The perception system is driven by registered depth and color images paired with an estimate of the RGB-D sensor's pose in the robot's coordinate frame. The dimensionality of the data processed by each stage is indicated in the figure, illustrating places where dimensionality reduction is used to improve efficiency.

a pose estimation of identifiable objects. The individual components of the perception pipeline are described in detail below.

\section{A. Conventions}

The robot coordinate frame is used to identify features of the system and the environment in the following discussion. The convention followed is that common to ROS: a righthanded coordinate system with the $\mathrm{X}$ axis extending forward from the robot, the $Y$ axis extending to the robot's left, and the $\mathrm{Z}$ axis extending up from the base. Objects may be positioned anywhere on the table surface, and are free to rotate about their vertical axes.

\section{B. Work Surface Extraction}

During execution, the table height, direction, and extents are periodically re-estimated from direct observation. This process is run every ten seconds to allow for robot movement during operation without requiring a reset of the system. In this stage, we search for a connected component of the depth image that is dominated by points within a narrow range of heights above ground level using a recursive histogram analysis.

The inliers of a successful analysis - consisting of a significant fraction of points within $2 \mathrm{~cm}$ of each other when projected onto the $\mathrm{Z}$ axis - are then used to extract the table orientation and extents which are used in subsequent object extraction. The table orientation is calculated as the eigenvector corresponding to the largest magnitude eigenvalue of the covariance matrix of the inlier points projected onto the $\mathrm{XY}$ plane. When a new table estimate is computed, it is atomically swapped into a memory cell referenced at the start of each new object detection update.

\section{Coarse Recognition and Localization}

Upon arrival of a pair of registered depth and color images, all points not within a space above the table geometry previously extracted are immediately removed. The remaining points are projected onto the line corresponding to the table direction, and clustered using a histogram analysis. These clusters are then run through a quick summarization procedure to determine if they could possibly be an object of interest. This check involves computing statistics regarding the HSV colors of the pixels corresponding to cluster points, and the overall spatial extents of the cluster. For instance, if a cluster has a vertical extent of only $10 \mathrm{~cm}$, it will not be identified as an object known to be $30 \mathrm{~cm}$ tall. Each object type of interest is therefore accompanied by constraints on allowable color and bounding geometry features.

Multiple coarse recognizers corresponding to the known object vocabulary may support any given cluster. All clusters recognized by at least one coarse recognizer have their centroids computed, and are fed forward in the system. When no coarse recognizer fires, this processing stage provides an opportunity for early exit.

\section{Refined Recognition and Localization}

The objects to be recognized and localized must be modeled before the system will identify them as anything other than unknown obstacles. A point cloud of an object's surface is first captured, then used to construct a truncated distance field representing a uniform sampling of a cost function whose minimal manifold corresponds to the surface of the object model. This sampling is computed by performing a Euclidean distance transform (EDT) on a voxel grid populated from the model point cloud. This preprocessing step is efficiently performed across multiple CPU cores using the approach described in [7].

Observed point clusters are recognized by finding a 2D transformation that minimizes this cost function when summed over all observed points. This approach is similar to [8], but the nonlinear optimization technique employed here is Powell's dogleg method [9]. The optimization procedure iteratively evaluates 2D transformations applied to 3D points whose transformed coordinates are used to index into the sampled cost function until a local minimum is found.

The necessary arithmetic and memory access patterns for this operation lie squarely within the core competencies of hardware designed for graphics acceleration. By loading the sampled cost function into a 3D texture in OpenGL, the cost of each transformed point is obtained by looking up a floating point value in a texture. The graphics processing unit (GPU) is used to perform linear interpolation along each axis of this texture lookup to further smooth the search space, and texture coordinates are clamped to truncate the cost function out to infinity, thereby mitigating the deleterious effects of outliers.

First, the image coordinates of the relevant points are translated to a cropped coordinate frame containing only the observed points. These coordinates are then paired with the $3 \mathrm{D}$ points reconstructed from the depth data, transformed into the object's coordinate frame using the points' centroid as the origin, and loaded onto the GPU. The 2D projection of the original data is used to address the output data as it 
is, by construction, an injective mapping from 3D points to $2 \mathrm{D}$ coordinates.

A GLSL vertex shader applies the candidate transformation, while passing through the $2 \mathrm{D}$ coordinates for each point unchanged. The fragment shader looks up the distance value from the cost function texture, and writes the interpolated value it arrives at to that point's location in the cropped 2D coordinate frame. Additionally, the optimization procedure lends itself to a batch interface, wherein several choices of parameters are evaluated in an overlapping fashion, thereby allowing the GPU to compute a cost function evaluation while asynchronously transferring the results of previous evaluations back to the CPU.

When the optimization process has finished, the average distance from each point in the observation under the minimal-cost transformation to the object model is considered to determine if we have a successful identification. Multiple optimizations may be performed to resolve the ambiguity of multiple coarse recognizers positively identifying point cluster summaries. An additional gain in efficiency is found by considering the rotational symmetry of each object. The general problem of identifying a 2D translation and $1 \mathrm{D}$ rotation is, for example, reduced to just the $2 \mathrm{D}$ translation optimization for objects that are fully symmetric. The rotational symmetry of each object is therefore recorded along with the object model information used by the coarse recognition step.

\section{E. Tracking}

The above process is applied to a small number of observed point clusters, while the rest are left as unidentified. All clusters are then passed to a tracking component that models the kinematics of both identified and unidentified point clouds. Observation integration is achieved by maintaining a track state that includes both object position and velocity in a Kalman filtering framework.

Inspired by the linear arrangement of objects on a long table, observations are aligned with existing estimates using the Needleman-Wunsch optimal matching algorithm [10]. This algorithm provides a principled way to match predictions of a linear chain of states to observations while taking potential object identification as well as position into account. In the usual application of this algorithm to string alignment, skip characters may be added at any point in one or the other strings. These are points at which two strings do not align, but whose removal from one string allows a likely match to continue beyond the missed location. The algorithm thus prevents us from aligning a track identified as one object with an observation identified as a different object type, and provides the necessary mechanism to inject new tracks and deal with data dropout.

The motion model used for tracking is one of zero acceleration. Furthermore, we are interested in the problem of tracking objects undergoing identical motion. We take advantage of this commonality by initializing the position covariance estimate of a new track by a sensor noise model as is usual, but carry over both velocity value and covariance from previous tracks. This causes the velocity estimate of successive tracks to adhere very tightly to a robust running average.

Final reporting from the tracker is governed by a configuration parameter indicating how many observations of any given track are needed before reporting is warranted. When a recognized object track is reported, a bounded history of orientation estimates provided by the localization optimization is passed through a RANSAC [11] procedure to compute a robust mean. The special consideration given to object yaw is due to the expectation that yaw measurements will be the noisiest axis of observation due to varying geometry occlusion as the view of an object changes over time. Consider, for example, a round watering can with a long spout. The yaw estimate for such an object will likely be much better when computed from views in which the spout is visible than those where it can not be seen.

\section{Manipulation}

When an object is detected, the pick-and-place manipulation pipeline gets called to pick up the approaching object and move it to a desired location. The manipulation pipeline is comprised of three main components, namely grasping, motion planning and execution. In this section we will discuss each component in depth. Before the pipeline is called, an arm is chosen to pick up the object. In our experiments, we are using a PR2 robot with two arms. Currently we are simply alternating between the arms. If no feasible grasps for the first arm are computed, we have found that quite often the other arm is capable of performing the pick-and-place action after receiving the next observation.

Note, that the same exact system is used for manipulating both static and dynamic objects. In the case of the static object, the entire pipeline just executes immediately, instead of waiting for the object to enter the robot's workspace.

\section{A. Grasping}

Reliable grasping of a static object by a robot's endeffector can be a difficult task given the uncertainty in each part of the system, from the object pose estimation to the robot's mechanical calibration. Additional problems arise when the object is moving at a fast speed. To simplify the grasp planning, we exploit the fact that we have a welldefined set of objects to be manipulated. This allows us to restrict the set of grasps for each type of object to ones that we have previously tested and saved in a database. Once an object is detected, the grasps are retrieved and checked for feasibility. We will now explain each component of the grasp selection process.

Types of Grasps. The process of picking up an object requires the end-effector to move between three different 6DOF poses:

- pregrasp - An end-effector pose that is offset from the object (see Figure 2, left). The motion planner is used to plan a collision free trajectory from the arm's waiting configuration to a valid configuration with the end-effector at the pregrasp pose. 


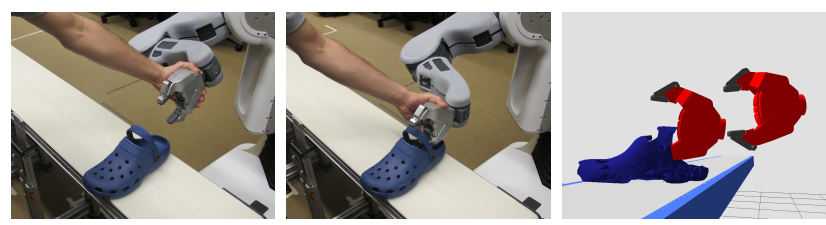

Fig. 2. From left to right, the user moves the end-effector to the pregrasp pose and then to the grasp pose. The pair of grasps is added to the database as they appear on the right.

- grasp - The pose of the end-effector in which its fingers are in position to reliably enclose part of the object (see Figure 2, middle).

- postgrasp - The end-effector pose after it moved away from the surface with the object grasped. The motion planner is then used to compute a feasible motion for the arm that takes the end-effector from the postgrasp to the desired place location.

Recording Grasps. Prior to manipulating a new object, we create a set of grasps for it. We found the grasp recording process to be simple and reliable. The process begins by placing the object within the workspace of either one of the arms and within the RGB-D sensor's view. Once the item is localized, the user is asked to move the end-effector to the desired pregrasp pose and the 6-DOF pose of the endeffector in the coordinate frame of the object is recorded. The process is repeated for the grasp pose. The pregrasp and grasp pair are then added to the set of grasps for that object type in the grasp database. In general, we found that a single set of postgrasp positions can be used for all object types so the user is not required to record a postgrasp for each object. Instead, the grasp orientation is paired with a set of postgrasp translations above the object for each object type. In our experiments, the set of grasps for each object contains between two and twenty pregrasp-grasp pairs. See Figure 2 for an example.

Grasp Selection. Upon detection of a new object, we use its estimated pose and velocity to predict $t_{\text {pred }}$ and $x_{\text {pred }}$. $t_{\text {pred }}$ is the time at which the object will be at the center of the workspace of the arm and $x_{\text {pred }}$ is the predicted 6-DOF object pose in the world. The object type is used to retrieve a set of pregrasps and grasps from the database. For each pregrasp, $p_{i}$, we use $x_{\text {pred }}$ to compute $x_{p_{i}}$, the pregrasp pose in the world frame for when the object will be at its predicted pose. The same is done for each grasp $g_{i}$ to compute $x_{g_{i}}$.

Before we add $\left\{x_{p_{i}}, x_{g_{i}}\right\}$ to $X_{p g}$, the set of feasible pregrasp-grasp tuples, the following two feasibility checks are performed on each tuple:

- chase grasps - It is very difficult to pick up a moving object using a grasp motion that is chasing it along the direction of its velocity, $v_{\text {object }}$. It rarely succeeds if $v_{\text {object }}$ is equal to or greater than the maximum speed of the end-effector along the grasp motion. Thus, we chose to filter out the chase grasps completely by detecting if the pregrasp to grasp motion is moving in the same direction as $v_{\text {object }}$. We do this by computing $c=\left\|v_{\text {object }}\right\| \cdot\left\|x_{g_{i}}\right\|$. If $c<k^{\circ}$, the tuple is deemed

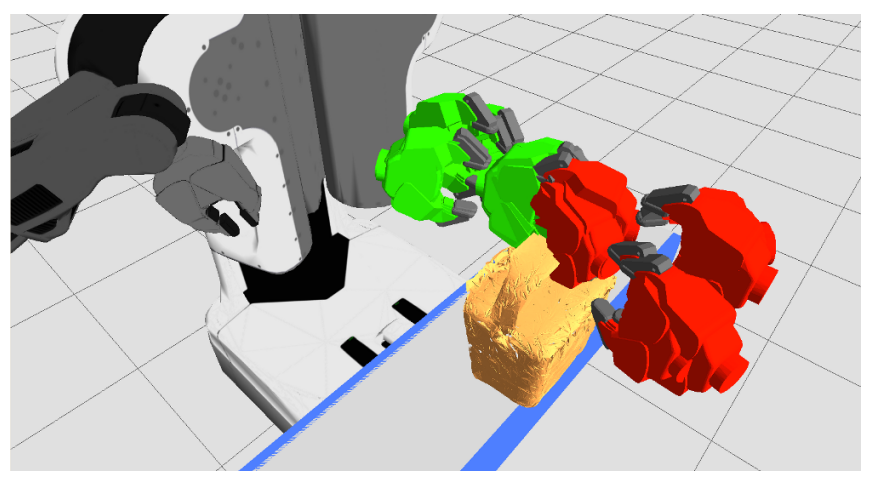

Fig. 3. The end-effectors in green are included in $X_{p g}$, while the ones shown in red are discarded by the kinematic feasibility check.

too similar to the object's velocity and it is rejected. In our experiments, we set $k$ to correspond to an angular difference of $-30^{\circ}$.

- kinematics - We check if each $x_{p_{i}}$ and $x_{g_{i}}$ is kinematically feasible for the arm of the robot using an inverse kinematics solver to compute the corresponding joint configuration. If either is invalid, the tuple is rejected. In Figure 3, six pregrasp-grasp tuples are shown for the basket. After they are checked for kinematic feasibility, the ones in red (darker color) are discarded because they are unreachable by the right arm.

Given a set of valid grasps, researchers often select the best grasp to plan to using some heurisics [12]. However, in our domain, this approach can be overly restrictive, as the feasibility of the grasp depends on the estimated time of arrival of the object. The better option is to let the planner itself figure out which grasp to plan to given the time constraint. Thus, instead of choosing a single pregraspgrasp tuple to target, the entire set of pregrasps in $X_{p g}$ is sent to the motion planner, allowing the planner to determine the $\left\{x_{p_{j}}, x_{g_{j}}\right\}$ in $X_{p g}$ that is feasible and optimizes costs well. After a plan is computed by the planner, the final waypoint of the planned path to the pregrasp, $x_{p_{j}}$, chosen by the planner. We use the corresponding grasp, $x_{g_{j}}$ as the grasp pose.

Computing the postgrasp is a straightforward process, generic across object types. Given the grasp, $x_{g_{j}}$, we compute the postgrasp, $x_{p o_{j}}$, by searching through the set of postgrasps for a kinematically feasible arm configuration that is as far from the table as possible. In our experiments, we found that the $x_{p o_{j}}$ chosen was typically within $12 \mathrm{~cm}$ of $x_{g_{j}}$.

\section{B. Motion Planning}

In an environment where robots work alongside people, it is important that the robot's behavior is consistent and predictable. This way, any human workers nearby can feel comfortable and safe around it. In particular, for a robot to be an effective member of a team on an assembly line that sorts moving objects into bins, the robot motions needs to be consistent, predictable and as close to optimal as possible in order for people to be able to work alongside and within the workspace of the robot. With this in mind, we implemented 
a heuristic search-based approach to generate motion plans for each of the robot's arms independently.

Heuristic searches such as $\mathrm{A}^{*}$ provide strong theoretical guarantees such as completeness and optimality or bounds on suboptimality [13]. Their generality allows for complex constraints and cost functions, while providing good cost minimization and consistency in the solution. Consistency here implies that given similar input, similar output is produced, thus making the robot's motions more predictable.

Motion planning for pick-and-place of moving objects needs to be performed as fast as possible so the robot has enough time to execute the computed motions in time. To combat the high dimensionality of the planning problem, we employ a heuristic search based approach that uses an anytime variant of $A^{*}$ called ARA* [14] that quickly finds an initial, and possibly sub-optimal, solution and repairs it while deliberation time allows. The approach also relies on a compact graph representation and informative heuristics to provide real time performance. Details on this approach and applications to single and dual-arm motion planning can be found in [15], [16].

We configured the planner to search in a 7 dimensional task space, $\{x, y, z$, roll, pitch, yaw, $\theta\}$, that represents the 6-DOF pose of the end-effector in the world frame, coupled with $\theta$, the position of the redundant degree of freedom in the robot's arm. This representation can be used when planning for a robot with one or more 7-DOF manipulators, such as the PR2 robot. In our experiments, we execute two independent instances of the planner, one for each arm. The cost function we use is aimed to minimize the 6-DOF path length of the end-effector.

During the pick action, the planner is called to plan a path from the waiting configuration of the arm to one of the pregrasps in $X_{p g}$. Before planning begins, the geometry of the fixed objects in the robot's workspace are added to the collision representation. In our experiments, this included the conveyor belt and bins that surrounded the robot. After the robot grasps the object, the collision geometry of the object is attached to the robot's collision model. During the place action, a plan is requested from the postgrasp to any one of the drop poses above the bins. Note that there is nothing constraining the user from having the robot gently place the item on a surface instead. Now that a potentially fragile object (or an object filled with something) is grasped in the robot's end-effector, we impose an upright path constraint on the planner when computing a path for the place action. The constraint requires the planner to maintain the initial $\{$ roll,pitch,yaw $\}$ of the end-effector throughout the path with a small tolerance in each dimension.

After a path is computed, we pass it through a simple deterministic shortcutting routine that can deal effectively with discretization artifacts. We found that only a single pass through the points was necessary. In our experiments, the entire shortcutting step lasted between 5-10ms, including checking the interpolated motions for collisions every $2^{\circ}$.

The ability to accurately predict the time it takes to execute a trajectory is essential to picking up a quickly moving object. Being that our motion planner plans solely for the kinematics of the arm, we then perform a final post-processing step, in which the shortest timing intervals between points are computed that enforce the robot's dynamic constraints [17]. The waypoint locations themselves are not moved, instead feasible velocities and accelerations are assigned. We found in our experiments that on average, for a given trajectory, the predicted execution timing and the actual trajectory execution timing differ by approximately $80 \mathrm{~ms}$.

\section{Execution}

Given that the object is moving at a reasonable pace, the pick action is substantially more difficult to execute than the place because very precise timing is needed to succesfully pick up the object with a firm grasp. For example, in our experiments the objects moved at approximately $33 \mathrm{~cm} / \mathrm{s}$ across the robot's workspace. At that velocity, if the execution of our grasp motion is $100 \mathrm{~ms}$ too late, the object will have passed $x_{\text {pred }}$ by more than $3 \mathrm{~cm}$, and, depending on the type of the object, it is very likely that the end-effector will swipe and miss.

Pick Action. At this stage in the pipeline, it is confirmed that the entire pick action is kinematically feasible. In the case of the pick action, the following two trajectories have been generated:

- $\operatorname{traj}_{p}$ - The pregrasp trajectory begins at the waiting configuration of the arm and ends at $x_{p_{j}}$. It is a collision free path generated by the motion planner, then shortcutted and filtered. $\operatorname{traj}_{p}$ has a predicted trajectory execution duration of $d_{p_{\text {pred }}}$ seconds.

- $\operatorname{traj}_{g}$ - The grasp trajectory begins with the wrist at $x_{p_{j}}$, then moves to $x_{g_{j}}$ and ends at $x_{p o_{j}}$. The path is an open loop motion in which the end-effector moves a very short total distance. $\operatorname{traj}_{g}$ is filtered to assure the robot can execute it. Note that we define $d_{g_{p r e d}}$ as the predicted trajectory execution duration of the trajectory from the start until $x_{g_{j}}$. We disregard the execution time from $x_{g_{j}}$ to $x_{p o_{j}}$ because the first half of the motion is the only time sensitive component, given that the endeffector has to be at $x_{g_{i}}$ to pick up the object at $x_{\text {pred }}$ at time $t_{\text {pred }}$.

The next step is to determine whether there is enough time for the robot to execute both trajectories in time to pick up the object at $t_{\text {pred }}$. The pickup is determined to be feasible if $t_{\text {pred }}>\left(t_{\text {now }}+d_{p_{\text {pred }}}+d_{g_{\text {pred }}}\right)$. If the pickup is feasible, then $\operatorname{traj}_{p}$ is executed immediatly. After it is completed, $\operatorname{traj}_{g}$ will begin execution at $t_{g_{\text {execute }}}=t_{\text {pred }}-$ $d_{g_{p r e d}}$. The end-effector is commanded to start closing as it is approaching the grasp pose. In our experiments, we found that depending on how early the object was detected and which arm is being used, the robot would sleep for between 0.0 and 1.5 seconds between executing the trajectories to ensure a well-timed grasp.

Place Action. After the object is grasped and raised to the postgrasp, there is not much work left to do. The computed 


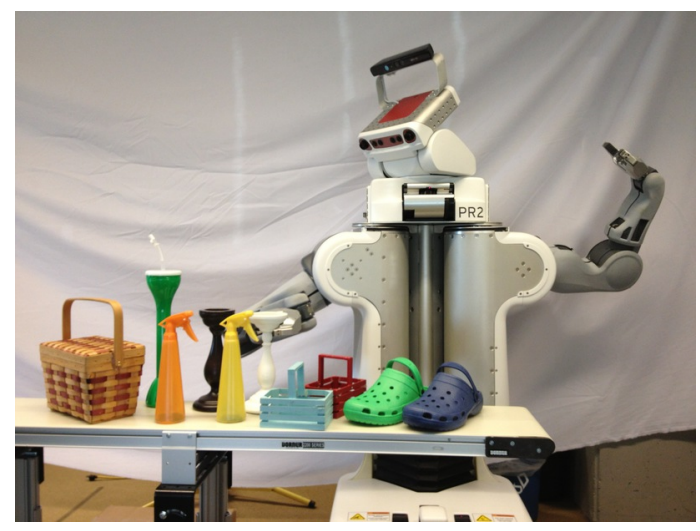

Fig. 4. The objects used for all experiments.

path with the upright orientation constraint on the endeffector is executed immediatly. After the end-effector opens, allowing the object to drop into the desired bin, the arm returns to the waiting configuration. The purpose of the waiting configuration is two fold. First, it is intended to keep the arm that is closer to the feed edge of the belt out of the view of the RGB-D sensor, regardless of where the bins are located. Second, it is desirable to keep the arm in a configuration that is close to the table to aid in quicker reaction times by having to execute shorter trajectories.

\section{EXPERIMENTS}

\section{A. Computing Platforms}

All logic related to planning was executed onboard the PR2's own computers. The perception software was hosted remotely on a desktop computer with an Intel Core i7-2600 quad-core $\mathrm{CPU}$ at $3.4 \mathrm{GHz}$ and an NVIDIA GeForce GT 420 GPU with 48 CUDA cores, a low-end graphics card from 2010. The imposition of networking between these two critical components adds a significant and variable amount of latency to the system, however the PR2 used for these experiments did not have a discrete GPU, and so could not host the perception system itself. The perception pipeline was written in the Haskell functional programming language, and compiled with the $\mathrm{GHC}^{1}$ 7.6.1 compiler.

\section{B. Object Selection}

The objects chosen, Figure 4, range in height from $15 \mathrm{~cm}$ to $45 \mathrm{~cm}$, and breadth from $8 \mathrm{~cm}$ to $25 \mathrm{~cm}$. These objects also display a variety of rotational symmetries, which affects their localization and how the objects may be grasped. The candlesticks and tall green bottle have an infinite order of rotational symmetry, denoted $C_{\infty}$, which translates to a freedom to grasp such an object from any angle, and frees the localization optimization from needing to consider object orientation. The shoe is not rotationally symmetric, denoted $C_{1}$, and must be grasped from its open end. The spritzer bottle is also $C_{1}$, but may be grasped from either side to hook the PR2's fingers underneath the overhanging geometry

\footnotetext{
${ }^{1}$ http: //www haskell. org/ghc
}

of the trigger mechanism. The various baskets are all $C_{2}$, as their handles may be grasped from either side.

Object models were acquired using RoboEarth software [18], a poster with fiducial markers, and a Kinect sensor. The models gathered for the experiments described here consist of 500 thousand to 1 million points.

\section{Pick-and-Place}

Figure 4 shows the conveyor belt used for testing both static grasps, in which objects are placed on the surface in front of the PR2 with the belt motor turned off, and dynamic grasps, in which the belt motor is on. In the static grasp configuration, the robot's head is oriented so that it is looking down at the table, and objects are rapidly placed in front of it. As soon as the robot begins to clear the work surface with an object in hand, a new object is placed on the surface. Objects are placed such that at least one arm can plausibly perform a grasp, but precise position and orientation are allowed to vary within that constraint.

The experiment conducted in the static grasp configuration involved 100 pick-and-place operations in which the robot removes an object placed onto the work surface in front of it, and places the object into one of two bins placed on either side of it. Of the 100 attempted actions, 91 were successful. The most common failure mode involved the object slipping out of the robot's gripper due to an insecure grasp. These 100 actions were timed in blocks of 10 , yielding an average of $6.7 \mathrm{~s}$ per pick-and-place action. During the time these experiments were conducted, the perception system failed to identify an object before a two second timeout period elapsed on two occasions. The experiments in which perception failed are not included in the reported time, as we did not have a consistent approach to failure recovery.

The dynamic configuration has the robot looking down the length of the conveyor belt, as in Figure 4. Objects are placed on the far end of the $2.13 \mathrm{~m}$ belt, and carried past the robot. In this configuration, the perception system only reports on objects it has seen a minimum of three times. This limits system responsiveness, but is important to eliminate spurious observations of the object being hand-placed on the end of the belt, and to ensure stability in pose estimation.

System performance was measured over 100 pick-andplace operation attempts with the belt at its top speed, $33 \mathrm{~cm} / \mathrm{s}$, 87 of which were successful. Six objects were effectively ignored due to the planner being unable to compute a suitable trajectory for either arm in the allotted time. Of these six, five were spritzer bottles, suggesting that the grasps chosen for this object did not leave the planner enough freedom to maneuver. The seven other failures were fumbled grasps. As in the static test, sometimes an insecure grasp would lead to an object being dropped. The dynamic test added the new failure mode of objects bouncing off of the back of the open gripper during a catch attempt. This contribution of momentum to the experiment was an excellent test of the system's overall timing: the gripper had to close around the object as it hooked available geometry in order to absorb all of its momentum without excessively destabilizing the 


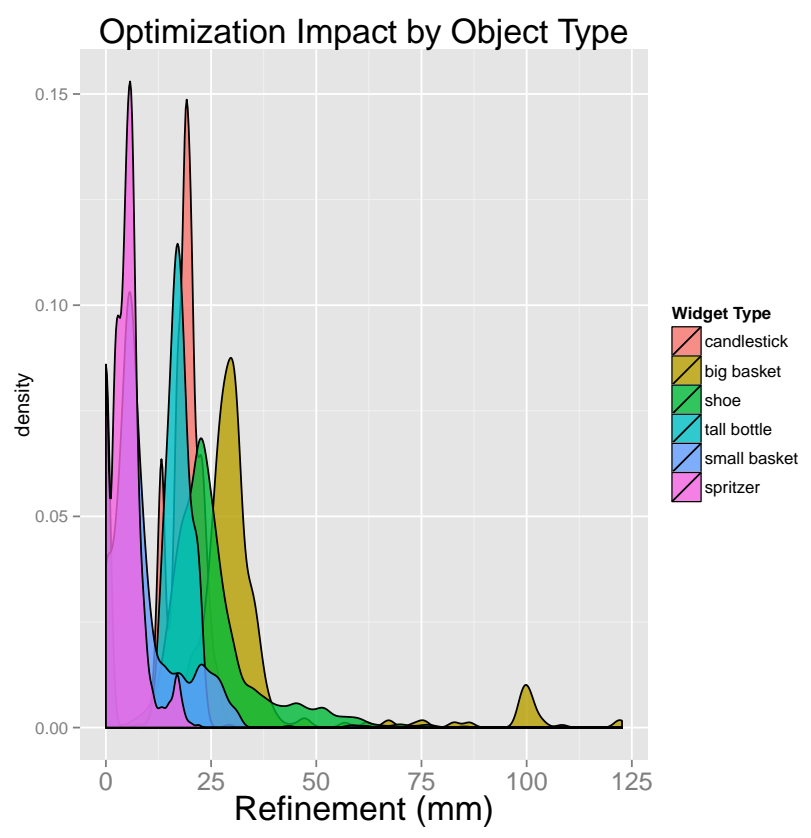

Fig. 5. A nonlinear optimization that fits observed points to a per-object cost function refines an initial localization estimate provided by each point cluster's centroid.

object. The tight timing constraints, paired with the design of the PR2's arms, meant that the robot's arm farther from where the objects were coming from was easier to utilize. Of the 94 attempted grasps, 57 were made by the far arm.

\section{ANALYSIS}

\section{A. Perception Performance}

Preprocessing includes collecting a point cloud for each object to be identified. The raw point data is used to populate a $0.5 \mathrm{~cm}^{3}$ resolution voxel grid, then passed through a Euclidean Distance Transform (EDT), taking an average of $304 \mathrm{~ms}$ for each object.

Once the system is running, parameters describing the conveyor belt surface are periodically recalculated as described in Section III-B. This process took an average of $39 \mathrm{~ms}$ per update, and was run concurrently with the rest of the perception pipeline in a separate thread.

The localization step begins by considering the centroid of each cluster of points found above the table surface. This is a reasonably good estimate of the object's position, but is biased towards the camera due to self-occlusion of the object geometry. The goal of navigating the PR2's finger into an opening with approximately $1 \mathrm{~cm}$ clearance on either side (e.g. underneath a spritzer bottle's trigger) demands a level of accuracy in object pose estimation which can be difficult to achieve when using only point cluster centroids. However, the localization refinement step represents a significant fraction of all the time spent in perception, so its utility is of interest. Recovering object yaw is critical for grasping asymmetric objects, but focusing solely on translational pose estimation reveals further contributions made by refining initial coarse position estimates.

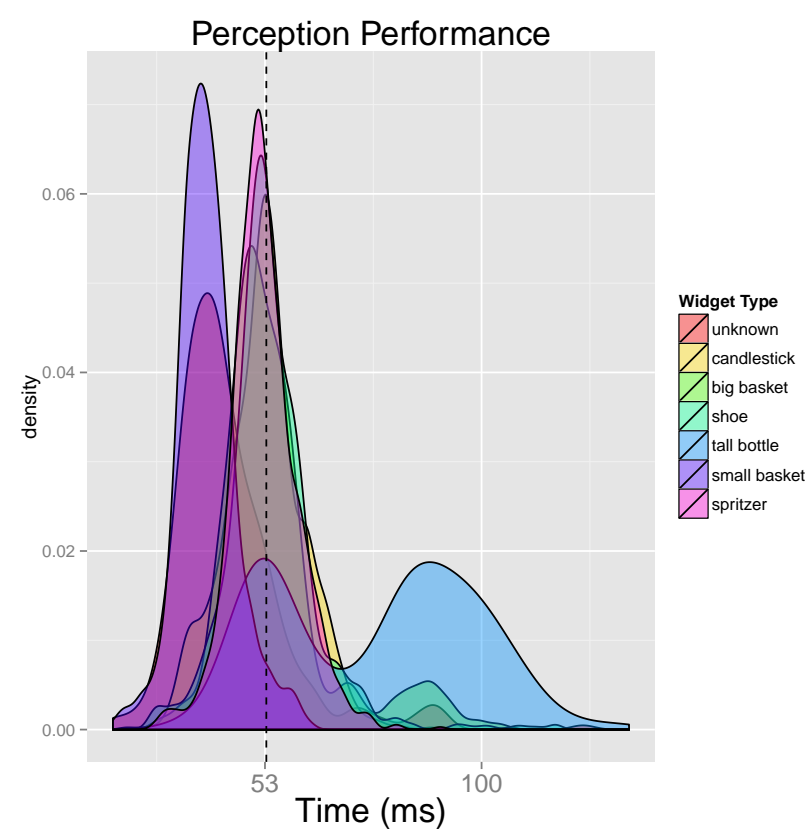

Fig. 6. Perception performance broken down by object type. The overall mean is $53 \mathrm{~ms}$.

Figure 5 illustrates the translation corrections made by the optimizer to an initial localization estimate based on point cluster centroids with a kernel density estimate of observed refinements. Note that in this run of experiments, there were no false identifications and no wild misses by the robot. The two object types needing the greatest correction from their initial centroid estimates were the big basket and the shoe, which are the two objects with the greatest asymmetry in the XY plane. The big basket is also a tall object, resulting in views looking down the table that primarily see the near side of the object. In contrast, most of the perimeter of an item like the small basket could be seen at the available view angle. This contrast is borne out by the effect of the optimization on these two objects: the big basket's translational position was shifted an average of $3.4 \mathrm{~cm}$ from the point cluster centroid, while the small basket was shifted an average of $0.9 \mathrm{~cm}$.

The average processing time for the perception system over the 5117 object detections during the moving object experiment was $53 \mathrm{~ms}$, Figure 6. The "tall bottle" object was the slowest to process, at an average of $77 \mathrm{~ms}$ per update. Initial filtering, clustering, and coarse recognition comprise the first perception phase, taking an average of $22.2 \mathrm{~ms}$. The second phase consists of the localization optimization procedure, taking an average of $31.1 \mathrm{~ms}$. The final tracking phase did not take a significant amount of time.

The average processing time for the motion planner during the moving object experiment was $182 \mathrm{~ms}$, including time spent in ROS service calls. This gives a minimum system response time on the order of $250 \mathrm{~ms}$ if only one observation of a scene is needed. For applications such as dynamic object manipulation, multiple observations may be needed, which 
pushes the delay before action to over $400 \mathrm{~ms}$. The performance of these experiments is thus completely dominated by the speed at which the PR2 can move its arms. The PR2 requires approximately $4.5 \mathrm{~s}$ to perform the motions needed for these tasks, which puts a lower limit on how quickly the entire system can process pick-and-place tasks.

\section{B. Robustness}

Execution time - a limiting factor in system responsiveness - was consistent throughout the experiments described. The longest single perception update in the dynamic test covering 5117 updates took $132 \mathrm{~ms}$. There are many sources of variance in these timings as the perception system was running on the GHC Haskell runtime system with its generational garbage collector, which itself was running in a desktop Linux environment (Ubuntu 10.04).

Localization accuracy was measured by considering the PR2's effective "hand-eye coordination." We used the PR2's proprioception to obtain an estimate of the location of one of the robot's fingertips with its arm extended over the work surface and angled down so that the finger just touched the surface. The location of this tangent was marked, and the arm was removed from the work area. A candidate object was then placed at the marked location, and the localization returned by the perception system was compared with the PR2's own estimate of where its finger had been.

These experiments yielded an average discrepancy between proprioception and perception of $5.3 \mathrm{~mm}$. A meter stick was then used to move the object one meter down the work surface from its starting location. The robot's head was turned to face this new location, and the output of perception was again recorded. This relative motion estimate yielded an average discrepancy between the meter stick and the perception system of $6.7 \mathrm{~mm}$.

The last metric recorded from the perception system was the speed of the belt. This was estimated during the experiment by tracking objects over time. The perception system estimated the belt speed at $33.1 \mathrm{~cm} / \mathrm{s}$, with a standard deviation of $0.08 \mathrm{~cm} / \mathrm{s}$. We were unable to obtain another measurement of belt speed with less noise.

The reported timings include all segmentation, recognition, and localization. There is additional overhead in the integrated system due to running the perception calculations remotely from the PR2 itself. The net result was a ROS object detection publisher rate that varied between 10 and $12 \mathrm{~Hz}$, the same rate at which raw depth images were received at the perception computer.

\section{CONCLUSION}

We have demonstrated pick-and-place operations performed by a PR2 at a rate of $6.7 \mathrm{~s}$ per object at a $91 \%$ success rate. Similar operations on a moving work surface yield an $87 \%$ success rate. Room for improvement remains in the areas of system integration, and end-effector customization. The speed at which the PR2's arms can move proved to be a limiting factor in system performance, and the gripper design was not particularly suited to absorbing the impact of moving objects. Despite these mechanical limitations, the PR2 proved to be capable of responsive, high throughput object manipulation.

\section{REFERENCES}

[1] J. A. Marvel, R. D. Eastman, G. S. Cheok, K. S. Saidi, T. Hong, E. R. Messina, B. Bollinger, P. Evans, J. Guthrie, E. Hershberger, C. Martinez, K. McNamara, and J. Wells, "Technology readiness levels for randomized bin picking," in Performance Metrics for Intelligent Systems (PerMIS), 2012.

[2] M.-Y. Liu, O. Tuzel, A. Veeraraghavan, Y. Taguchi, T. K. Marks, and R. Chellappa, "Fast object localization and pose estimation in heavy clutter for robotic bin picking," The International Journal of Robotics Research, 2012

[3] C. Papazov, S. Haddadin, S. Parusel, K. Krieger, and D. Burschka, "Rigid 3d geometry matching for grasping of known objects in cluttered scenes," International Journal of Robotics Research, vol. 31, pp. 538-553, 2012.

[4] O. Birbach, U. Frese, and B. Bäuml, "Realtime perception for catching a flying ball with a mobile humanoid," in IEEE International Conference on Robotics and Automation (ICRA), 2011, pp. 5955-5962.

[5] S. Chitta, E. G. Jones, M. Ciocarlie, and K. Hsiao, "Perception, planning, and execution for mobile manipulation in unstructured environments," IEEE Robotics and Automation Magazine, Special Issue on Mobile Manipulation, vol. 19, 2012.

[6] R. Wagner, U. Frese, and B. Bäuml, "3d modeling, distance and gradient computation for motion planning: A direct gpgpu approach," in Proceedings of the IEEE International Conference on Robotics and Automation (ICRA 2013), 2013.

[7] A. Meijster, J. Roerdink, and W. Hesselink, "A general algorithm for computing distance transforms in linear time," Mathematical Morphology and its applications to image and signal processing, pp. 331-340, 2002.

[8] C. Papazov and D. Burschka, "Stochastic global optimization for robust point set registration," Computer Vision and Image Understanding, vol. 115, no. 12, pp. 1598-1609, Dec. 2011.

[9] M. J. D. Powell, "An efficient method for finding the minimum of a function of several variables without calculating derivatives," Computer Journal, vol. 7, no. 2, pp. 155-162, 1964.

[10] S. B. Needleman and C. D. Wunsch, "A general method applicable to the search for similarities in the amino acid sequence of two proteins," Journal of molecular biology, vol. 48, no. 3, pp. 443-453, Mar. 1970.

[11] M. A. Fischler and R. C. Bolles, "Random sample consensus: a paradigm for model fitting with applications to image analysis and automated cartography," Commun. ACM, vol. 24, no. 6, pp. 381-395, June 1981. [Online]. Available: http://doi.acm.org/10.1145/358669. 358692

[12] K. Hsiao, S. Chitta, M. Ciocarlie, and E. Jones, "Contact-reactive grasping of objects with partial shape information," in Intelligent Robots and Systems (IROS), 2010 IEEE/RSJ International Conference on, Oct., pp. 1228-1235.

[13] J. Pearl, Heuristics: Intelligent Search Strategies for Computer Problem Solving. Addison-Wesley, 1984.

[14] M. Likhachev, G. Gordon, and S. Thrun, "ARA*: Anytime A* with provable bounds on sub-optimality," in Advances in Neural Information Processing Systems (NIPS) 16. Cambridge, MA: MIT Press, 2003

[15] B. J. Cohen, G. Subramanian, S. Chitta, and M. Likhachev, "Planning for Manipulation with Adaptive Motion Primitives," in Proceedings of the IEEE International Conference on Robotics and Automation (ICRA), 2011

[16] B. Cohen, S. Chitta, and M. Likhachev, "Search-based planning for dual-arm manipulation with upright orientation constraints," in Proceedings of the IEEE International Conference on Robotics and Automation (ICRA). St. Paul, Minnesota: IEEE, 2012.

[17] K. Anderson, "Smoother and more efficient trajectory planning," July 2012. [Online]. Available: http://www.willowgarage.com/blog/2012/ 07/13/smoother-and-more-efficient-trajectory-planning

[18] D. Di Marco, A. Koch, O. Zweigle, K. Häussermann, B. Schiessle, P. Levi, D. Gálvez-López, L. Riazuelo, J. Civera, J. M. M. Montiel, M. Tenorth, A. Perzylo, M. Waibel, and R. van de Molengraft, "Creating and using roboearth object models," in IEEE International Conference on Robotics and Automation (ICRA), 2012, pp. 35493550 . 